\title{
Diseño metodológico para la investigación de las rutas de estudio y aprendizaje. Reporte de investigación
}

\author{
An Integrated Methodological design for the \\ research of the study paths and learning.
} -Final Report-.

Diana Gil Chaves ${ }^{1}$

Neila Sánchez ${ }^{2}$

\section{Resumen}

¿Qué aspectos se pueden tener en cuenta en el momento de definir un diseño metodológico para abordar las rutas de estudio y aprendizaje de los estudiantes de grado séptimo de varias escuelas de Bogotá, cuando trabajan el tema de proporcionalidad en el aula de clase? Pero sobre todo, ¿con qué instrumentos de indagación se puede contribuir para el análisis de la complejidad del aula de clase? Responder las anteriores preguntas es el propósito fundamental del presente documento, producto de una experiencia investigativa de varios años, en el proceso de construcción de la metodología como parte del proceso mismo de investigación.

\begin{abstract}
What aspects can be considered when definite methodologies approach the paths at study and learning of seventh grade students in several schools in Bogota? Even, taking in to account the proportionality in the class room? What research instruments can contribute to complexity analysis in the class room? Answering the previous questions is the main intention at this document. It's the product of a several year research experience in the methodology building as a part of the research process. These analyses are based on the complexity of the class room's culture.
\end{abstract}

\section{Palabras clave:}

Investigación calitativa, triangulación, fiabilidad, observación

Key words:

Qualitative Research, Triangulation, Reliability, Observation

1 Licenciada en Psicología y Pedagogía de la UPN, Mg. En estructuras y Procesos del Aprendizaje de la U. Externado de Colombia. Docente de la Licenciatura en Educación Básica con Énfasis en Matemáticas, de la Facultad de Ciencias y Educación, Universidad Distrital. Investigadora del Grupo de Investigación CRISALIDA. dgil@udistrital.edu.co

2 Licenciada en Matemáticas de la Universidad Distrital, Especialista en computación para la docencia Universidad Antonio Nariño Docentes de la Licenciatura en Educación Básica con Énfasis en Matemáticas, de la Facultad de Ciencias y Educación, Universidad Distrital. Investigadoras del Grupo de Investigación CRISALIDA. sanchez.neila@gmail.com 


\section{Introducción}

El presente documento busca, dar cuenta del proceso de construcción de la fundamentación metodológica para el desarrollo de la investigación "Matemáticas y su aprendizaje en la educación básica del Distrito Capital. Caracterización de las rutas de estudio y aprendizaje en el aula".

Para el cumplimiento de este propósito el documento se estructura de la siguiente forma, en la primera parte se realiza la presentación del marco institucional dentro del cual se encuentra la presente investigación; en la segunda parte hace referencia a la justificación del diseño metodológico utilizado; en la tercera parte se presenta una caracterización del diseño metodológico; en la cuarta se hace alusión al proceso de diseño y pilotaje de cada uno de los instrumentos de indagación y en la quinta parte de este escrito se presentan los instrumentos definitivos para la recolección de información del ambiente de la clase de un grupo de estudiantes de grado séptimo de varios colegios oficiales de Bogotá, en el momento en que abordan el tema de la proporcionalidad inversa.

\section{Presentación}

El proyecto de investigación denominado: "Matemáticas y su aprendizaje en la educación básica del Distrito Capital. Caracterización de las rutas de estudio y aprendizaje en el aula", hace parte de los trabajos desarrollados por el grupo de investigación CRISALIDA, del proyecto curricular de Licenciatura en Educación Básica con énfasis en Matemáticas. El grupo de investigación está conformado por docentes del proyecto curricular LEBEM, como investigador principal tenemos a Jorge Orlando Lurduy Ortegón y como coinvestigadores: Neila Sánchez Heredia, Pedro Gerardo Rocha Salamanca, Diana Gil Chaves y Fernando Guerrero Recalde, además de la participación permanente de los estudiantes de la Licenciatura en Matemáticas y la Licenciatura en Educación Básica con énfasis en Matemáticas, como pasantes de investigación en las diferentes fases de desarrollo de ésta. La participación en la investigación, de los estudiantes y los docentes del
Proyecto Curricular, ha permitido a partir de la inmersión dentro del proyecto de investigación en el aula, hacer un aporte significativo a la formación de docentes investigadores.

Dicho proyecto de investigación se desarrolla en dos etapas: la primera denominada "La construcción teórica y metodológica de la investigación sobre rutas de aprendizaje", implementada en tres fases: fase I. Construcción teórica y metodológica de la propuesta de investigación 2002-2003; fase II. Desarrollo de la investigación 2004 I y fase III. Análisis de los resultados 2004 II - 2005 I. La segunda etapa, denominada "Aplicación metodológica de la investigación" implementada en dos fases: Fase I de recolección, organización y análisis de la información 2005 II y 2006 I y II y la fase II. La elaboración de conclusiones y del informe final 2007 I. En este contexto, el presente documento es un resumen que corresponde a la fase II y la fase III, de la primera etapa del proyecto de investigación, en cuanto a lo relacionado con: la construcción teórica de la perspectiva metodológica, diseño, validación y pilotaje de los instrumentos de indagación.

Puesto que, la realización de todo trabajo de investigación en el campo educativo y específicamente en el área de la educación matemática, necesariamente tiene que dedicar tiempo importante a la indagación, búsqueda y análisis de la metodología a ser implementada para el buen desarrollo de la investigación; este trabajo tiene como propósito mostrar el proceso de construcción de las bases teóricas del diseño metodológico para el proyecto de investigación "Matemáticas y su aprendizaje en la educación básica del Distrito Capital. Caracterización de las rutas de estudio y aprendizaje en el aula", con el fin de hacer aportes importantes a otras propuestas similares, además de generar la discusión académica en torno a este tema tan importante dentro de todo trabajo de investigación en el aula.

\section{Justificación de una metodología cualitati- va para la investigación}

Para el caso particular de la investigación "Matemáticas y su aprendizaje en la educación básica del 
Distrito Capital. Caracterización de las rutas de estudio y aprendizaje en el aula" la definición de la metodología, las técnicas, el diseño de los instrumentos y las estrategias de implementación, han obedecido a un proceso que inicia desde la elaboración de un estado del arte ${ }^{3}$, en el cual se estudiaron las principales metodologías y los instrumentos de investigación utilizados para dar cuenta del aprendizaje y comprensión de conocimientos matemáticos puestos en juego en el espacio escolar y más específicamente en el aula de clase.

Esta revisión documental dio aportes significativos en varios sentidos: uno en cuanto a la formación de los estudiantes del Proyecto Curricular de la Licenciatura en Matemáticas y los de Licenciatura en Educación Básica con énfasis en Matemáticas, puesto que hubo participación de cinco estudiantes quienes apoyaron la búsqueda documental, distribuidos en tres grupos de trabajo en las siguientes temáticas:

- "Un estado del arte sobre metodologías cualitativas de investigación - en educación- desde la perspectiva de la didáctica de la Matemática en torno a la estructura multiplicativa" desarrollada por los estudiantes Diana Milena Florido y Diego Alejandro Silva.

- "Estado del arte sobre instrumentos cualitativos de indagación para la investigación en el aula. Una mirada desde la Educación Matemática" desarrollado por las estudiantes Diana Paola Castro Valencia y Mónica Liliana Bernal Vaca.

- "Estado del arte de los instrumentos metodológicos utilizados en las investigaciones sobre comprensión en Matemáticas" desarrollado por William Vargas Ricaurte.

La participación de cada uno de los estudiantes en esta pasantía, les permitió conocer de forma activa el desarrollo de procesos de investigación documental, en cuanto a la búsqueda y clasificación

3 Ver síntesis de esta indagación en: Metodología de investigación, enfoques, técnicas e instrumentos para el análisis y sistematización de información sobre la comprensión de información sobre la comprensión en Educación Matemáticas. En: Cuadernos de Investigación. Número 5, IEIE Universidad Distrital Francisco José de Caldas. 2.005. de información, elaboración de fichas ${ }^{4}$ - reseña bibliográfica, descriptiva, sinóptica, cuantitativa y comprensión teórica global- y la sistematización-informe, sobre las indagaciones realizadas, de esta forma los estudiantes tuvieron la posibilidad de iniciarse en este tipo de actividades y por otra parte, de apropiarse del referente conceptual de las diferentes temáticas abordadas. Esto se constituyó en un importante aporte a la formación de los estudiantes para profesor del proyecto curricular de la Licenciatura en Matemáticas, en la medida en que los enfrenta con algunas de las actividades propias de la investigación en el campo de la Educación Matemática y además les permite reconocer la importancia de la investigación en este campo.

La revisión documental, plasmada en cada uno de los estados del arte elaborados, permitió conocer y evidenciar el momento en el cual se encuentra la investigación en Educación Matemática, en particular la escasa investigación en el aula, referente a las acciones y actividades que realizan los estudiantes en el momento de abordar el tema de proporcionalidad inversa en las clases de Matemáticas de séptimo grado.

A continuación se hará una presentación de cada uno de los aportes de la revisión documental, para la definición de algunos aspectos conceptuales de lo metodológico para la presente investigación.

\section{En cuanto a la construcción de la perspecti- va metodológica}

Cuando el grupo de trabajo se enfrentó a realizar el diseño metodológico para la investigación, se encontró con diversos problemas; el primero se pudo evidenciar dentro del desarrollo de la primera etapa en la Fase II denominada estado del arte y tiene que ver con el hecho que dentro del medio, aun son muy escasas las investigaciones que tienen como objeto de estudio la ruta o trayectoria de aprendizaje, lo cual, implica desde el punto de vista meto-

4 Para el desarrollo metodológico de los estados del arte se tuvieron en cuenta, los planteamientos de Hoyos B., C (2000). Un Modelo para Investigación Documental., Medellín: Editorial Señal editora,. 
dológico, gran dificultad para encontrar un marco teórico unificado (Hoyos, 2000).

Otra de las dificultades y a su vez características del diseño metodológico es la naturaleza del objeto de estudio, puesto que, la ruta o trayectoria de estudio es emergente, es decir, sólo es posible describirla y analizarla desde la observación y análisis de diferentes estrategias metodológicas de las acciones de los estudiantes dentro del ambiente y las interacciones propias del aula de clase, cuando abordan problemas de proporcionalidad inversa o directa. A partir de reconocer que el aula de clase tiene unas condiciones que no permiten la eliminación de las diferentes fuentes de variación que afectan al objeto de estudio, lo cual implica que los resultados no pretenden en ningún momento ser generalizables a una población mayor a la observa$\mathrm{da}$, pero si, tiene la posibilidad de ser replicada en otros espacios educativos.

Esto se diferencia de algunas propuestas de investigación en el aula, donde se definen el diseño metodológico a partir de la elaboración de hipótesis, que son luego, verificadas o refutadas en las indagaciones en el aula, con el propósito de generalizar los resultados de la misma.

Todo lo anterior llevó a la necesidad de definir la perspectiva desde la cual se realizará esta investigación, es decir, especificar las particularidades y las características del proceso metodológico que orientaría la investigación. Esto implica reconocer las diversas posiciones al respecto, Shulman (1986), nombra dos polos extremos: El enfoque positivista o proceso producto, que tiene como intención encontrar leyes y de confirmar hipótesis y el enfoque interpretativo, orientado más a la búsqueda del significado. Mientras que, Goetz y LeCompte (1988), consideran que en general, muchas de las actuales investigaciones educativas, en especial los diseños más creativos, pueden catalogarse en un punto intermedio entre ambos paradigmas y plantean el modelo de investigación a partir de cuatro dimensiones o modos suposicionales: deductivoinductivo, generativo-verificativo, constructivoenumerativo y subjetivo-objetivo.
La perspectiva metodológica de la presente investigación, se enmarca dentro de una dimensión diferente a las anteriores, reconocida por Godino (2003), como la dimensión socio-critica, entendida esta como la posibilidad de conectar la investigación con la práctica, con la vista puesta hacia su cambio en la dirección de una mayor libertad y autonomía de los participantes. Puesto que como lo plantea Kilpatrick (1988), no es suficiente penetrar en una clase y observar el encuentro educacional. Se requiere además de la colaboración entre el profesor y el investigador.

Además, define como método de indagación la utilización de las técnicas cuantitativas, puesto que, como lo reconoce Lorenzo Blanco (1991), entre las dos perspectivas existe "complementariedad y necesariedad" (Alvira, 1983, p 58), al contrario de otras tesis que afirman la imposibilidad de su apoyo mutuo. Sin embargo, esta superación de la incompatibilidad de los métodos no es óbice para que algunos autores hablen de los "obstáculos en el empleo conjunto de métodos cualitativos y cuantitativos" (Reichardt y Cook, 1986). En consecuencia, como lo afirma Blanco (1991, p 4) parece más propio que se comience a hablar de complementariedad de las dos perspectivas, aún cuando seamos conscientes de las dificultades que tal unión pueda plantear.

Dentro de la presente investigación, es claro que no se pretende realizar generalizaciones, ni comprobar hipótesis formuladas con anterioridad a la observación de la ruta. Puesto que se reconoce que "el método es obra de un ser inteligente que ensaya estrategias para responder a las incertidumbres. En este sentido, reducir el método a programa es creer que existe una forma a priori para eliminar la incertidumbre. Método es, por lo tanto, aquello que sirve para aprender y a la vez es aprendizaje... No existe un método fuera de las condiciones en las que se encuentra el sujeto" (Morin, 2003, p 32). Por tanto, la pretensión está en diseñar y formular una(s) estrategia(s) metodológica(s) que permitan dar cuenta de la ruta de estudio y aprendizaje, que emerge de las observaciones a las acciones realizadas por los estudiantes cuando solucionan o resuelven problemas relacionados con la proporción 
directa o inversa en el aula de clase de grado séptimo; teniendo en cuenta las diferentes interacciones -entre los estudiantes, los profesores y el entorno- que permiten la manifestación, descripción, comprensión y análisis de esta trayectoria.

\section{En cuanto a la relación entre el objeto y el método.}

Un aporte importante de la búsqueda documental, es la discusión entorno a la relación entre el diseño metodológico y el objeto de estudio particular de la investigación, lo cual implica alejarnos de la concepción tradicional de objetividad y acercarnos en su lugar a una nueva "concepción según la cual un sistema, en lugar de ser una estructura definible como una realidad separada del sujeto que la considera, es una entidad definible justamente en su relación con ese sujeto." Es decir, el estudio y análisis de un sistema es considerado como una realidad compleja, en la que es necesario tener en cuenta además de ella, al sujeto que se propone conocerla, puesto que, un sistema adquiere características reflexivas, al resultar constituido por la reflexión recíproca de la actividad de sujeto sobre la actividad del sistema presuntamente objetivo por él definido." (Navarro, 1990, p 51)

Estas características, implican necesariamente reconocer la existencia de interferencias -efectos reflexivos - entre la actividad del sistema objeto y la actividad del sujeto para conocerlo. La interferencia, entendida como la coexistencia de varios dominios de objetivación, en el sentido de reconocer la existencia de procesos de objetividad y al mismo tiempo procesos de subjetividad como partes importantes y necesarias en la construcción de sentido de la realidad, puesto que los mismos no son ni mutuamente reducibles ni completamente separables. Es decir, requiere reconocer que el aula de clase, es una realidad compleja y requiere para su descripción integrar distintos niveles de análisis como son: las transformaciones de los esquemas de pensamiento, las acciones y las producciones de los estudiantes, las interpretaciones y descripciones de los actores de lo que acontece en el aula y por supuesto de los observadores externos.

\section{En cuanto a la construcción de los instrumentos.}

Otro fundamental aporte del estado del arte, es todo lo relacionado con el diseño de instrumentos de indagación en el aula de clase, lo cual determinó, la definición de la observación directa como la técnica principal, para la recolección de información utilizada, entendida según Jean-Marie de Ketele (1995) como "un proceso que incluye la atención voluntaria y la inteligencia, orientadas por un objeto para obtener de él información." Dentro de las siguientes características:

- Se debe privilegiar las manifestaciones y acciones observables del comportamiento de los estudiantes en el aula de clase, cuando se enfrentan al problema de desarrollar pensamiento multiplicativo.

- Se reconoce que el lugar privilegiado para la observación de la ruta de estudio es el aula de clase, a manera de hipótesis. Lo cual implica, que para entender el significado de los hechos que ocurren en el aula de clase de septimo grado en el momento de abordar el tema de la proporcionalidad, se realice desde una perspectiva compleja, el tratamiento y análisis de los datos.

- Por la naturaleza de la investigación se tiene en cuenta la interacción entre los diferentes actores que intervienen en la manifestación de la trayectoria; los estudiantes, los profesores y el entorno, elementos a tener en cuenta al describir, comprender, analizar y construir la ruta de estudio más probable.

- Se consideró importante orientar y dirigir la gestión y organización del trabajo de los profesores en el aula, a partir del diseño, formulación y validación de secuencias didácticas en proporcionalidad directa e inversa, mediante un proceso sistemático. Además de realizar una inducción a los profesores en torno a qué acciones debían privilegiar en el momento de implementar la secuencia de actividades en el grado séptimo en los Colegios Rodrigo Lara Bonilla, República de Panamá y Federico García Lorca. 


\section{En cuanto a la definición y delimitación de los polos a ser estudiados.}

Otro aporte significativo, consistió en definir que la investigación retomaría el tetraedro didáctico (Lurduy, 2009) como una herramienta de investigación para el análisis de los procesos de enseñanza-aprendizaje en el aula de clase, y que se constituiría en la representación del sistema didáctico - estudiante, saber, profesor y entorno- puesto que, permite visualizar, comprender y representar los componentes y las relaciones que se establecen entre los mismos. Además de definir el lado correspondiente al que esta investigación hace énfasis, señalando que lo relevante, es analizar la relaciòn entre el polo cognitivo, es decir, las actuaciones cognitivas de los estudiantes cuando se enfrentan a las actividades propuestas por el profesor para tratar el tema de proporcionalidad, polo didáctico, lo relacionado con el diseño de las actividades por parte del profesor, para lo cual se realizará el diseño de la secuencia de actividades y del polo ecológico relacionado con la cultura y las interacciones sociales del aula.

\section{En cuanto al aprendizaje}

El estado del arte permitió, conocer diversas posturas frente a cómo entender el aprendizaje y a su vez la forma de investigarlo, la más usual es la de tener dos grupos de estudiantes uno de ellos un grupo control y el otro al cual se le aplica o implementa un diseño de actividades donde de antemano se esperan unos resultados determinados, que luego serán contrastados con las pruebas que se les aplican a los estudiantes para verificar el aprendizaje previamente presupuestado y así poder hacer la diferencia con el grupo control. Es decir, se considera desde esta postura que hubo aprendizaje en el estudiante si este es capaz de realizar adecuadamente las pruebas control que se le colocan.

Dicha postura mecanicista del aprendizaje, poco adecuada para dar cuenta del polo cognitivo, parte importante de la presente investigación, generó que se planteara una concepción constructivista del aprendizaje, donde primero es necesario situar un campo conceptual y utilizar la noción de esque- $\mathrm{ma}^{5}$ para poder dar cuenta del aprendizaje de los niños cuando estudian algunos aspectos del campo conceptual multiplicativo; puesto que, aquí se parte de comparar los esquemas de pensamiento que se ponen en juego en el momento de resolver inicialmente una actividad -momento $\mathrm{A}-$, con esquemas en dos momentos posteriores, uno durante el proceso de desarrollo e implementación de la secuencia de actividades - momento $\mathrm{B}-\mathrm{y}$ otro al final del proceso - momento $\mathrm{A}^{\prime}-\mathrm{y}$ sólo se considera que hubo aprendizaje si se evidencia modificación y transformación en los esquemas de pensamiento en el momento de resolver las diferentes situaciones que se plantean en cada uno de estos momentos.

\section{Características de la metodología utilizada}

\section{Investigación Cualitativa}

El tipo de investigación en la cual nos encontramos es cualitativa y etnográfica en general, específicamente es una investigación de tipo interpretativo, exploratorio y descriptivo, que busca explicitar la ruta de estudio de los estudiantes a partir del análisis del polo cognitivo, con la teoría del interaccionismo simbólico, cubriendo de esta manera los cuatro polos del tetraedro didáctico, enfatizando en el plano estudiante - saber - entorno.

Una investigación cualitativa es una actividad sistemática orientada a la comprensión en profundidad de fenómenos educativos y sociales, a la transformación de prácticas y escenarios socioeducativos, a la toma de decisiones y también hacia el descubrimiento y desarrollo de un cuerpo organizado de conocimientos. Carr y Kemmis, (1988).

Además, una forma de desarrollar una investigación cualitativa es mediante la investigación etno-

5 Retomando a Vergnaud "Un esquema es una totalidad organizada, que permite generar una clase de conductas diferentes en función de las características particulares de cada una de la situaciones de la clase a la cual se dirige y comporta los siguientes componentes: invariantes operatorios - conceptos-en-acto y teoremas-en-acto-, anticipaciones del fin a lograr, reglas de acción e inferencias -o razonamientos-". Ibíd. P. 104 
gráfica, la cual busca interpretar, explorar y describir la vida, las costumbres, creencias, prácticas y comportamientos de un ethnos. Según Carr y Kemmis (1988), "una investigación cualitativa es una actividad sistemática orientada a la comprensión en profundidad de fenómenos educativos y sociales, a la transformación de prácticas y escenarios socioeducativos, a la toma de decisiones y también hacia el descubrimiento y desarrollo de un cuerpo organizado de conocimientos".

Para Goetz y LeCompte (1988), las fases que integran el proceso de investigación etnográfica son: la primera fase constituye el período previo al trabajo de campo, se determinan las cuestiones de la investigación y los marcos teóricos preliminares y se selecciona un grupo para su estudio. La segunda fase plantea el acceso del investigador al escenario, la selección de informantes y fuentes de datos, así como decisiones en torno al tipo de estrategias de recolección de información y tipos de registro para su almacenamiento. La tercera fase nos remite al trabajo de campo propiamente dicho. La cuarta fase se centra en el análisis intensivo de la información. Aspectos que se pueden ver reflejados en el proceso de desarrollo de la presente investigación.

\section{Pensamiento complejo}

- Una de las características fundamentales de la presente investigación es el reconocimiento de un pensamiento complejo, para el estudio, análisis y comprensión de la realidad del aula de clase, puesto que, "pensar de forma compleja es pertinente allí donde — casi siempre - nos encontramos con la necesidad de articular, relacionar, contextualizar. Esto sólo es posible sí retomamos algunos de los principios para pensar de forma compleja, propuestos por Morin (2003), entre los que encontramos los siguientes:

- El principio sistémico u organizacional, se tiene en cuenta en el proceso metodológico en el momento que se reconoce el sistema didáctico y el tetraedro didáctico como herramienta fundamental dentro del proceso metodológico.
- El principio hologramático: al igual que en un holograma cada parte contiene la totalidad de la información del objeto representado y a su vez el todo se encuentra en la parte. Esto se relaciona con el proceso metodológico de la investigación, puesto que, para la elaboración de los instrumentos de investigación se tuvo en cuenta que cada uno de los instrumentos de indagación proporcionará elementos, que permitieran a los investigadores hacer la descripción de la ruta de estudio que siguen los estudiantes a partir de cruzar y relacionar la información que cada uno proporciona.

- El principio de retroactividad: consiste en que al romper con el concepto de causalidad lineal, la causa no sólo actua sobre el efecto, sino que a su vez el efecto retroactua sobre la causa permitiendo la autonomía organizacional del sistema. Este principio se relaciona directamente con la investigación, en el momento en que para la descripción de la ruta de estudio, se aplica el concepto de bucle tetralógico, para el análisis de las múltiples relaciones que hacen parte del tetraedro, puesto que, es preciso concebir cada uno de los polos en conjunto, es decir, como términos a la vez complementarios, concurrentes y antagónicos.

- El principio de recursividad: un proceso recursivo es aquel cuyos productos son necesarios para la propia producción del proceso. Esto se evidencia en el reconocimiento de la realidad compleja del aula, en la cual, cada polo del tetraedro didáctico requiere de lo que sucede o mejor produce cada uno para el funcionamiento del otro polo, es decir se establece una dinámica auto-productiva y autoorganizacional al interior del sistema didáctico.

- El principo dialógico: puede ser definido como la asociación compleja -complementaria/ concurrente/antagonista - de instancias necesarias, conjuntamente indespensables para la existencia, el funcionamiento y el desarrollo de un fenómeno organizado. Este principio es esencial para el desarrollo metodológico de la presente investigación, puesto que, la importancia, relación, complementariedad y 
función de cada uno de los polos - didáctico, cognitivo, epistémico y ecológico- permite percibir la complejidad de la realidad del aula, dentro de la cual emerge la ruta de estudio de los estudiantes.

- El pricipio de reintroducción: reconoce que el papel del sujeto observador es importante como constructor de conocimiento, en la medida en que el sujeto construye la realidad por medio de los principios antes mencionados. (Morin, et al. (2003). pp. 37-42).

\section{Interaccionismo simbólico}

Como la ruta de estudio se presenta en el entorno del aula de clase, la teoría del interaccionismo simbólico orienta la metodología de la investigación, ya que este, como perspectiva teórica metodológica de investigación social, la asunción con Blumer de que el investigador debe situarse dentro del proceso de definición del actor para comprender su acción central. Así el investigador debe ver el mundo desde el punto de vista de los sujetos que estudia:

Debemos ser capaz de tomar el rol de otros. Esta toma de rol es una interacción, es una interacción simbólica porque es posible solo por los "símbolos significativos", esto es el lenguaje y otras herramientas simbólicas, que los seres humanos compartimos y a través de los cuales nos comunicamos. Solo a través del diálogo podemos ser conscientes de las percepciones, sentimientos y actitudes de los demás e interpretar sus significados. De ahí el término interaccionismo simbólico (Crotty, 1998).

Para Blumer (1969), el interaccionismo simbólico presenta tres premisas:

- Los seres humanos actúan respecto de las cosas, e incluso respecto de las otras personas.

- la atribución de significados a los objetos mediante símbolos es un proceso social continuo. Los significados son productos sociales que surgen durante la interacción.

- Los actores sociales asignan significados a situaciones, a otras personas, a las cosas y así mismos a través de un proceso de interpretación.

Y tiene dos pasos distintos:

- El actor se indica a sí mismo que las cosas tienen significado.

- En función de un proceso de comunicación consigo mismo, la interpretación se convierte en una cuestión de manipular significados.

Según Godino (2003), el interaccionismo simbólico es una perspectiva teórica que tiene implicaciones analíticas y que ha sido utilizada para estudiar las relaciones entre las características sociales de los procesos de interacción, así como las existentes entre el pensamiento del profesor y el de los estudiantes. Cuyo supuesto básico es que las dimensiones culturales y sociales no son condiciones periféricas del aprendizaje matemático sino parte intrínseca del mismo.

Se enfatiza como foco de estudio las interacciones entre individuos dentro de una cultura en lugar de sobre el individuo. El énfasis se coloca en la construcción subjetiva del conocimiento a través de la interacción, asumiendo el supuesto básico de que los procesos culturales y sociales son parte integrante de la actividad matemática.

Para caracterizar el interaccionismo simbólico en educación matemática se describe su posicionamiento en relación a:

- El significado, la naturaleza del conocimiento matemático y los procesos de llegar a conocer -Comprensión Matemática-.

- El papel del lenguaje.

- El aprendizaje.

El Enfoque Ontológico Semiótico de la cognición e Instrucción Matemática propone el postulado del Relativismo epistemológico retomado en la presente investigación, puesto que este postulado "obliga a pensar en el conocimiento, el significado y la comprensión como nociones relativas y siempre 
determinables de manera parcial.”6 Además lleva a considerar el problema de la validez de los estudios investigativos, desde la perspectiva relativista, multicomponente y parcialmente determinable.

La triangulación de la información y sus procesos de fiabilidad

Para esta investigación se tuvo en cuenta la triangulación como la posibilidad de emplear numerosas técnicas de recolección de información, para tener la posibilidad de complementariedad entre ellas y de esta forma permitir a los etnógrafos determinar la exactitud de sus conclusiones a partir del cruce, efectuando triangulaciones con varias fuentes de datos. Las triangulaciones impiden que acepte demasiado fácil la validez de su impresión inicial; amplia el ámbito, densidad y claridad de los constructos desarrollados en el curso de la investigación, y ayuda a corregir los sesgos que aparecen cuando el fenómeno es examinado por un solo observador.

La presente investigación retoma los planteamientos de Goetz \& Lecompte (1988), cuando propone aplicar al trabajo de los etnógrafos unos fundamentos de fiabilidad (Martínez, 2000, la denomina confiabilidad) y validez usados en corrientes positivistas, acomodándoles en la perspectiva cualitativa, etnográfica y fenomenológica, es así, como aborda los problemas de la fiabilidad, entendida como la capacidad que tiene un estudio de replicarse. Es decir, que si un investigador usa los mismos métodos de un estudio determinado llega a los mismos resultados, pero para esta investigación se tiene en cuenta la fiabilidad desde las ciencias humanas, donde se reconoce que es imposible reproducir en condiciones exactas el comportamiento humano y por ende su estudio. Por lo tanto, la fiabilidad es entendida como el nivel de concordancia interpretativa entre diferentes observadores y evaluadores. La fiabilidad puede ser de dos tipos:

Fiabilidad externa: cuando investigadores independientes, al estudiar una realidad en tiempos o

6 "Es un postulado por el" Teoría de las funciones Semióticas. Un enfoque ontológico-semiótico de la cognición e instrucción matemática. Noviembre de 2003. situaciones diferentes, llegan a los mismos resultados, hay confiabilidad cuando varios observadores, al estudiar la misma realidad concuerdan con sus conclusiones.

Para alcanzar un buen nivel de confiabilidad externa, Goetz \& Lecompte (1984), aconsejan recurrir entre otras a las siguientes estrategias:

- Precisar el nivel de participación y la posición asumida por el investigador en el grupo estudiado.

- Identificar claramente a los informantes. Estos pueden representar grupos definidos y dar información parcial o prejuiciada.

- Describir detalladamente el contexto en el que se recaban los datos: contexto físico, social e interpersonal.

- Identificar los supuestos y meta teorías que subyacen en la elección de la terminología en los métodos de análisis para que sea posible una cierta réplica de la investigación

- Precisar los métodos de recolección de la información y de su análisis, de tal manera que otros investigadores puedan servirse del in forme original como de un manual de operación para repetir el estudio.

La fiabilidad interna: Se refiere al grado de consenso entre diferentes observadores de la misma realidad, elevar la credibilidad que merecen las estructuras significativas descubiertas en un determinado ambiente, así como la seguridad de que el nivel de congruencia de los fenómenos en estudio es fuerte y sólido.

Para reducir las amenazas que se le presentan a la confiabilidad interna se pueden utilizar varias estrategias:

- Usar categorías descriptivas de bajo nivel de inferencia, es decir, lo más concretas y precisas posible para que los datos sean primarios y frescos.

- El mejor aval para la confiabilidad interna es el trabajo en equipo, pues garantiza un mejor equilibrio de observaciones, análisis e inter- 
pretaciones. En algunos casos, las investigaciones son realizadas por un equipo cuyos componentes reciben con autoridad una extensa formación y que debaten el significado de las observaciones efectuadas hasta alcanzar un acuerdo.

- Utilizar todos los medios técnicos disponibles en la actualidad para conservar en vivo la realidad presenciada — grabaciones de audio y de video, fotografías entre otros-, de tal manera que pueda ser realizada y analizada por otros investigadores o por los evaluadores de la investigación. Estos instrumentos refuerzan la fiabilidad de los resultados.

\section{La observación y sus formas de registro}

Teniendo en cuenta que según Paul Croll (1995), el procedimiento más utilizado para dirigir una observación sistemática de clase es la observación en vivo donde el investigador registra lo que sucede en la clase, a menudo ayudado de algún dispositivo para medir el tiempo. El proceso de observar y registrar es virtualmente simultáneo. El investigador una vez terminado el periodo de observación, no dispone de ninguna ayuda mecánica para ayudarle a reconstruir lo que aconteció y no confía en la memoria para escribir una descripción de ello.

La ventaja de este procedimiento es que apenas perturba el desarrollo de la clase y que cuenta con la flexibilidad de un observador humano. Un único observador o, a veces, un par de observadores pueden pasar casi desapercibidos en la mayoría de las situaciones de clase y puede dirigir su atención a aquellos individuos y sucesos que lo requieran, adaptándose rápidamente a los cambios con objeto de responder a lo que esta ocurriendo. Los problemas que lleva el uso de un sistema de observación vivo son, por un lado, que los acontecimientos observados sólo pueden recrearse en términos del sistema de observación utilizado y, por otro, que la naturaleza del sistema de observación sistemática está limitada por la necesidad de que el observador observe y registre en el tiempo. Todo aquello que no forme parte del sistema de observación sistemático no puede por tanto recuperarse en forma alguna tras el periodo de observación.
Existe un límite en cuanto a la complejidad del sistema de observación cuando se dan observación y registro. Un enfoque alternativo a la observación en vivo es la utilización de una cámara de vídeo, alternativa tenida en cuenta para la presente investigación. Puesto que tiene las siguientes ventajas: Se puede someter una cinta de video de una clase, o de una parte de la misma, a un análisis mucho más complejo y flexible de lo que es posible hacer con una observación en vivo. El investigador puede ver la cinta tantas veces como desee, puede detenerse allá donde requira tomar notas o considerar dentro de qué categoría codificar un determinados suceso, y puede regresar a dicho punto siempre que albergue la más mínima duda en cuanto a la idoneidad de su decisión.

Asimismo puede cambiar y desarrollar el sistema de observación durante el periodo de análisis y no tiene por qué decidir los detalles del sistema hasta haber visto los datos. Al contrario, un observador que realice una observación en vivo, habrá tirado por la borda el trabajo que haya llevado a cabo hasta entonces si descubre que el programa utilizado no funciona u omite algún aspecto de interés. Una ventaja adicional del video es que el material obtenido pueden verlo varios observadores. Esto puede facilitar la adopción de un enfoque multifacético del problema de investigación o puede implicar la misma utilización del material para distintos propósitos.

A pesar de todas estas ventajas, la investigación reconoce los diversos problemas derivados de la falta de experiencia en el uso de dichas técnicas. Estos problemas se deben a las dificultades prácticas y a aquellas otras asociadas a la idoneidad de la visión de las clases que emergen de dichos sistemas. Las dificultades prácticas más obvias son aquellas relativas a los recursos y a las dificultades físicas para realizar la grabación. Grabar una clase requiere mucha organización y perturba mucho más el desarrollo de la misma que la presencia de un observador. Una dificultad adicional se deriva de la idoneidad de la visión que surja de la grabación de la clase.

En todo estudio observacional existe el problema de la influencia del propio proceso de observación sobre el desarrollo de lo que acontece. La agresivi- 
dad del equipo y la asociación a "salir en televisión" que el procedimiento conlleva, coloca al investigador en peligro de influir sobre la situación objeto de observación que cualquier otro que observa en vivo. Otro aspecto que puede ser derivado de dicho sistema de observación tiene que ver con el hecho que una cámara de vídeo carece de la flexibilidad del observador humano, tanto en términos de rapidez para dirigir su atención de un punto a otro de la clase, como de capacidad para observar la totalidad de la clase de un vistazo al tiempo que se mantiene el centro de atención en un determinado acontecimiento.

La utilización del vídeo como sistema de observación se ha puesto en práctica en aquellos casos en los que los intereses de la investigación han sido muy específicos o con objeto de proporcionar retroalimentación a profesores en cursos de formación. Este procedimiento más que como fuente de datos, se ha utilizado para ayudar a desarrollar sistemas de observación y en la formación de observadores.

Construcción de los instrumentos finales de indagación

Esta fase comenzó en el segundo semestre del año 2005, a continuación se presentan los cuatro instrumentos ${ }^{7}$ finales, resultado del diseño y rediseño del grupo de profesores investigadores, con los cuales se realizó el proceso de recolección de la información.

\section{Dinámica general de clase. Perspectiva} del profesor

Este instrumento consiste en una entrevista realizada a los profesores al finalizar cada actividad, su propósito es desarrollar una descripción pormenorizada de la clase realizada $-\mathrm{u}$ observada- que permita la identificación de los acontecimientos significativos del aula; enfatizando en los diferentes polos: polo epistémico, polo ecológico, polo didáctico y polo cognitivo, describien-

7 o de co-investigadores de la etapa 1 fase 3 , fue el encargado de rediseñar estos instrumentos, pero los aplicados en este momento son el resultado del diseño y rediseño del grupo de profesores investigadores. do la relación de los mismos. También se tiene en cuenta las diferentes relaciones que se encuentran en el aula como lo es profesor-estudiante, profesor-saber, estudiante-saber, estudiante-entorno, profesor-entorno entre otras, y en los análisis de la dinámica del hecho educativo, caracterizando las acciones que generan la ruta de aprendizaje.

Dado que éste instrumento es una entrevista semiestructurada, compartimos con Canell, C. y Cahn (1968), la definición de entrevista de investigación como "un diálogo iniciado por el entrevistador con el propósito específico de obtener información relevante para la investigación y enfocado por él sobre el contenido especificado por los objetivos de investigación, de descripción, de predicción o de explicación sistemáticas". Es un método inusual que comprende la reunión de datos a través de una interacción oral directa entre individuos.

\section{Interacción en el aula- roles y organización}

El objetivo de este instrumento es describir las interacciones o relaciones lógico abstractas que presentan los estudiantes cuando estudian problemas de proporcionalidad inversa junto con los diferentes roles dados dentro del aula, el papel que juega el profesor dentro del aula - gestión-, el rol desempeñado por los instrumentos o recursos didácticos implementados en cada actividad - manipulación de los recursos-. Además se describen los diferentes elementos físicos sensoriales presentes en el aula, la distribución del mobiliario, el cómo se emplea el espacio, y los diferentes fenómenos dados al respecto.

Este instrumento se aplica en el desarrollo de cada actividad y tiene por objeto describir las diferentes interacciones o relaciones lógico abstractas que presentan los estudiantes en el aula cuando se abordan actividades de razón y proporción, además se describe el rol del profesor explicando cada una de las acciones que realiza al momento de orientar el trabajo de los estudiantes. 
También se describe el papel que juegan los instrumentos o recursos didácticos utilizados para el desarrollo de la actividad y cómo es su utilización. En esta parte se explica, qué es lo que realizan los estudiantes en el momento de manipular los recursos. Se describen los diferentes elementos físicos sensoriales presentes en el aula durante el desarrollo de la actividad, como el espacio y la distribución de los pupitres de los estudiantes, además si ésta se altera al momento de la utilización del material o del trabajo realizado en grupo.

\section{Instrumento de portafolio. Trabajo individual del estudiante}

Este instrumento se aplica para cada actividad, y como su nombre lo indica por cada estudiante. Su propósito es establecer las manifestaciones de conducta cognitiva que se daban en los estudiantes cuando resolvían las situaciones propuestas durante clase para caracterizar la ruta de estudio seguida por los estudiantes.

Se utilizó el instrumento con el cual se hace una categorización de las respuestas de los estudiantes, en unos niveles creados bajo la teoría de Gerard Vergnaud (1990). La importancia de los instrumentos de análisis de dichos momentos radica en la comparación, para poder establecer un cambio de manifestaciones de conducta cognitiva y así concluir en qué condiciones comenzó y terminó la ruta de estudio de los estudiantes al abordar problemas de proporcionalidad inversa.

Las categorías que se tienen en cuenta en este instrumento tienen subcategorías que permiten evidenciar el desempeño del estudiante en la misma. El instrumento cuenta con unos cuantificadores -Realiza Completamente, Realiza Satisfactoriamente, Realiza Parcialmente, No lo Realiza y Otro- ${ }^{8}$, además con una descripción cualitativa detallada del porque de la escogencia del cuantificador.

La primera categoría es "aceptación del compromiso educativo, adopción de una actitud positiva

8 Categorización realizada por los profesores que pertenecen al grupo de la investigación. hacia el estudio y de cooperación con los compañeros", en ella se hace énfasis en el compromiso de los estudiantes frente a la actividad y de la actitud que presentaron durante la misma.

La segunda categoría corresponde a "exploración, indagación, búsqueda de conjeturas y modos de responder a las cuestiones planteadas", en ella se describe si el estudiante dio muestras de haber realizado una consulta extra-clase, la cual le ayuda para resolver la situación planteada, y además de esto se tiene en cuenta si el estudiante creó estrategias de solución y si reflexionó sobre las mismas.

La tercera categoría da cuenta de "recuerdo, interpretación y seguimiento de reglas - conceptos y proposiciones - y del significado de los elementos lingüísticos en cada situación", en esta se describe cuál es la utilización del lenguaje escrito y oral del estudiante al momento de explicar o argumentar sus estrategias de solución ya sea en la guía, a sus compañeros o durante la socialización de las estrategias.

En la cuarta categoría se tuvo en cuenta la "formulación/comunicación de soluciones a las situaciones o tareas propuestas, ya sea al profesor, a toda la clase o en el seno del grupo", se describe en ella la actitud frente al saber que el estudiante evidencia en el desarrollo de la clase, ya sea participando activamente en la socialización, proponiendo estrategias relevantes, aportando nuevas cuestiones y puntos de vista frente a soluciones dadas y en general aportando para llegar a una solución colectiva.

La quinta categoría "argumentación y justificación de conjeturas - al profesor o los compañeros-" refiere a las argumentaciones orales y escritas que cada estudiante propone, si puede relacionar los conceptos previos con la estrategia de solución y si utiliza un lenguaje claro para explicarla y justificarla.

En la sexta categoría: "recepción de información sobre modos de hacer, describir, nombrar, validar" se describe la actitud del estudiante frente a 
las sugerencias del profesor y las explicaciones en las discusiones con sus compañeros.

La séptima categoría "demanda información: estados en los que los estudiantes piden información al profesor o a otros compañeros" se describe los procesos que siguen los estudiantes al sentir incapacidad para interpretar la situaciones a resolver y las acciones a realizar.

\section{Los procesos de interacción}

En cada sesión se empleó este instrumento, este tiene por objeto identificar las diferentes interacciones que se dan al desarrollar una actividad, entre estudiante-estudiante, estudiante-saber, estudiante-maestro, estudiante-entorno. En este se considera la participación de los estudiantes a la hora de enfrentar la situación propuesta, en cuanto a sus explicaciones, búsqueda de estrategias individuales o colectivas de solución, el empleo de los recursos didácticos, el tipo de trabajo realizado - escritos, verbales-, las discusiones dadas entre ellos, entre los estudiantes y el maestro, entre otros.

El objetivo de este instrumento era identificar las diferentes interacciones que daban lugar al desarrollo de la actividad, las explicaciones de los estudiantes, el uso de recursos didácticos donde se describía la utilización del material por parte de los estudiantes y se decía qué tan apropiado o no, fue. Iniciativas personales y estrategias de solución individual relevantes, se identificaban cuáles eran las principales estrategias que proporcionaban aportes significativos para la construcción social del conocimiento sobre la proporcionalidad inversa, realizada en la socialización.

\section{Organización y sistematización de la información}

Al recoger la información a través de estrategias no interactivas como la observación no participante por medio del instrumento portafolio y de estrategias interactivas como la entrevista, se hizo necesario organizar y sistematizar dichos registros escritos.
Este material, trascripción de las entrevistas, el instrumento individual de cada estudiante "portafolio", las grabaciones, para que sea lo más completo y detallado, pasó por un proceso de categorización o clasificación, que exigió un esfuerzo mental, que implicó revisar los relatos escritos - respuestas escritas dadas por los estudiantes en cada guía-, ver los videos en varias ocasiones, primero, con la actitud de revivir la realidad en su situación concreta y, después, con la actitud de reflexionar acerca de la situación vivida para comprender lo que pasa.

Cada nueva revisión del material escrito, audición de los diálogos o visión de las escenas filmadas nos permitió captar aspectos o realidades nuevas, detalles, matices no vistos con anterioridad, acciones que enriquecieron el significado. En la práctica, en cada revisión del material fue necesario hacer anotaciones marginales que permitieron describir lo que se estaba interpretando.

La categorización hecha hasta aquí puede haber consistido en poner marginalmente algunos rótulos de categorías y algunas propiedades o atributos de estas categorías, como también en hacer diferentes tipos de anotaciones referidas a algunos aspectos de las grabaciones.

Ahora se trata de categorizar o clasificar las partes en relación con el todo, de describir categorías o clases significativas, de ir constantemente diseñando y rediseñando, integrando y reintegrando el todo y las partes, a medida que se revisa el material y va emergiendo el significado de cada hecho o dato.

En un principio el significado se ve de manera implícita, luego se va haciendo explícito con la aparición de símbolos verbales - categorías-, ahora bien, todo símbolo verbal o categoría aspira a representar a su referente, pero no hay símbolo que sea capaz de describir todos los rasgos del referente; en consecuencia, está obligado a omitir uno o varios de ellos. Por eso, todo símbolo es abstracto en sus representaciones de la naturaleza, pierde algo o mucho de ella y no es estrictamente adecuado o representativo. 


\section{Reflexiones finales}

El presente trabajo se enmarca dentro de los procesos investigativos desarrollados por uno de los grupos de investigación, que hace parte de la Licenciatura en Educación Básica con énfasis en Matemáticas (LEBEM). Por tanto, apoya la propuesta de formación en cuando integra a los estudiantes para profesor a los procesos investigativos de docentes más avanzados, que buscan hacer transformaciones pedagógicas desde el reconocimiento de la realidad educativa de las instituciones escolares de la educación básica y media, facilitando la formación de un docente investigador y reflexivo de su propia práctica.

Otra relación con la propuesta de formación LEBEM se encuentra, cuando se presenta el diseño metodológico del proyecto de investigación: investigación "Matemáticas y su aprendizaje en la educación básica del Distrito Capital. Caracterización de las rutas de estudio y aprendizaje en el aula", en el que se evidencia la necesidad y la posibilidad de realizar el estudio desde el reconocimiento de la complejidad del aula de clase, a partir del sistema didáctico y su mirada sobre cada uno de los polos y las relaciones existentes entre el polo didáctico, el polo cognoscitivo y el polo ecológico.

Además la presente propuesta de investigación permite evidenciar la relación entre algunos planteamientos teóricos con realizaciones en el aula de clase. Parte importante, puesto que dentro de la Licenciatura somos conscientes que en la formación profesional no es suficiente que el estudiante para profesor conozca, estudie y sepa sobre las diferentes teorías desarrolladas por disciplinas relacionadas con la educación sino que es indispensable propiciar espacios para que ellos integren, interactúen, teoricen, desarrollen, apliquen y gestionen dichos constructos teóricos en su realización como profesional de la educación, dando solución a los problemas del profesor. Lorenzo Blanco (1991), los denomina componente estática: que es el conocimiento teórico. Y el componente dinámica: que es el conocimiento que responde al saber hacer.

\section{Bibliografía}

Antillan, R. (1991). ¿Cómo entendemos la Sistematización desde una concepción metodológica dialéctica? Guadalajara: IMDEC-ALFORJA.

Balderrama, M. (1994). Investigación en educación (La Entrevista). En: La investigación etnográfica aplicada la educación. Dimensión educativa. Quito: Corporación Ecuatoriana de Investigación y Servicios Educativos.

Batanaz, L. (1998). Organización Escolar. Bases cientificas para el desarrollo de las instituciones educativas. Universidad de Córdoba: Servicio de Publicaciones.

Bauersfeld, H. (1995). "Language Games" in the mathematics classroom: their function and 20 their effects. En: Cobb, P. y Bauerselfeld, $\mathrm{H}$. (Eds). The Emergence of Meaning: Interaction in Class-room Cultures. Hillsdale, N. J.: Lawrence Erlbaum Associates, Pub.

Bejarano, C. (2000). Variables físicas del entorno escolar e influencias en el rendimiento académico. En: Opciones pedagógicas. 21: 132. Bogotá.

Blanco, L. (1991). Apuntes sobre metodología cualitativa. Badajoz: Universidad de Extremadura.

Blumer, H. (1969). Symbolic Interactionism: Perspective a method. Englewood Cliffs, N.J.: Prentice-Hall.

Botero, C. (2000): Un modelo para la investigación. Guía teórico-práctica sobre construcción de Estados del Arte. Medellín: Ed. Señal.

Briceño, D., Serrano, L. y Pulecio D. (2006). Informe final de pasantía de investigación: Matemáticas y su aprendizaje en la educación básica del distrito capital. Caractización de las rutas de aprendizaje en el aula pilotaje y validación de instrumentos de instrumentos de recolección de información. Bogotá: Universidad Distrital Francisco José de Caldas. 
Briones, G. (1995). La Investigación en el Aula y en la Escuela. Bogotá: SECAB:

Brousseau, G. (1986). Fundamentos y métodos de la didáctica de las matemáticas. Recherches En: Didactique De Mathe Matique. 7. (2): 33115.

Cáceres, J. (1998). Técnicas de Investigación en Sociedad, Cultura y Comunicación. México: Eds. Prentice Hall, Pearson Educación y Addison Wesley.

Canell, C. y Kahn, R. (1968). The dynamics of interviewing. New York: John Wiley \& Sons Inc.

Cárdenas, J., Quiroga, J. y Escamilla, A. (2004). Informe final pasantía de investigación: Matemáticas y su aprendizaje en la educación básica en el distrito capital. Caracterización de las rutas de aprendizaje en el aula. Bogotá.

Carr, W. \& Kemmis, S. (1988). Teoría crítica de la enseñanza. La investigación-acción en la formación del profesorado. Barcelona: Martínez Roca.

Cascallana, M. (1988). Iniciación a la matemática. Barcelona: Santillana.

Chamorro, C. (2003). Didácticas de las Matemáticas para primaria. Madrid: Ed. Pearson Prencite Hall.

Chevallard, Y. (1991). La Transposition DidactiqueDu savior savant au savior enseigné. Grenoble: La penssé sauvagé.

Cohen, L. y Manion, L. (1990). Métodos de investigación educativa. Madrid: Ed. La Muralla.

Coll, C. (1986). Acción, interacción y construcción del conocimiento en situaciones educativas. En: Revista de Educación. 279.

Coll, C. y Gómez, C. (1994). De qué hablamos cuando hablamos de constructivismo. En: Alegría de enseñar. 18.
Croll, P. (1995). La Observación Sistemática en el Aula de Clase. Madrid: Ed. La Muralla.

Crotty, M. (1998). The foundations of social science research. St. Leonards, New South Wales: Allen \& Unwin.

Davis, G. y Tall, D. (2002). ¿Qué es un esquema? Intelligence, Learning and Understanding - A tribute a Richard Skemp. UK: Universidad de Southtamptom \& Universidad de Warwick.

De ketele, J. y Rogers, C. (1995). Metodología para la recogida de información. Madrid, España. Ed. española: Editorial La Muralla, S. A.

Doménech y Viñas (1999). La organización del espacio y del tiempo en el centro educativo. Barcelona, España: Graó.

Dubinsky, E. (1991). Reflective abstraction in advanced mathematical thinking. Tall, D., (Ed.). Advanced Mathematical Thinking, Reidel, Londres.

Dueñas, M., Hernández, L. y Prieto, O. (2005). Informe final de pasantía de investigación: Aplicación y validación de instrumentos. Condiciones que posibilitan el estudio de las rutas de aprendizaje. Bogotá: Universidad Distrital Francisco José de Caldas.

Felicity, H. (2002). Ética y escuela, ¿Es siempre ético cumplir las normas de la escuela? Barcelona: $1^{\text {a }}$ edición, Editorial Gedisa S.A.

Fiol, M. (1990). El análisis didáctico y teórico de la proporcionalidad. Madrid, España.

Fiol, M. y Fortuny. (1990). Proporcionalidad directa. La forma y el número. Madrid: Síntesis.

Florez, R. \& Tobón, A. (2001). Investigación educativa y pedagógica. Colombia: Ed. Mc. Graw Hill.

Gairin, J. (1990). Las actitudes hacia las matemáticas. En: Actitudes hacia la educación. Barcelona: Boixareu Universitaria.

REVISTA CIENTÍFICA / ENERO -DICIEMBRE DE 2009 / No. 11 / BOGOTÁ, D.C. 
Godino, J. (2003). Teoría de las Funciones Semióticas en Didáctica de las Matemáticas. Un enfoque ontológico semiótico de la cognición e instrucción matemática. Departamento de Didáctica de la Matemática. Facultad de Ciencias de la Educación. Universidad de Granada. Acceso: 19 de marzo del 2005. Disponible en: http://www.ugr.es/local/jgodino/. Granada,España.

Godino, J. (2003). Teoría de las funciones Semióticas, Un enfoque ontológico- semiótico de la cognición e instrucción matemática. Acceso: 19 de marzo del 2005. Disponible en: http: // www.ugr.es/local/jgodino/.

Godino, J. y Llinares, S. (2000) Interaccionismo Simbólico en la educación matemática. En: Revista educación matemática. 12. (1): 70-92.

Goetz, J. y LeCompte, M. (1988). Etnografía y diseno cualitativo de investigación educativa. Madrid: Morata

Grupo DECA. (1992). Orientaciones para el diseño y elaboración de actividades de aprendizaje y de evaluación. En: Aula. 6: 33-39.

Grupo MESCUD. (2002). Matemáticas para todos. El sentido de la profesión profesor(a) de matemáticas. Bogotá: Fondo de publicaciones Universidad Distrital.

Hammersley, M. y Atkinskon, P. (1994). Etnografía: Métodos de investigación, Barcelona: Paidós.

Hoyos, C. (2000). Un modelo para Investigación Documental. Guía teórica y práctica sobre construcción de estados del arte. Bogotá: Editorial Señal.

Lurduy, et al. (2004-2007). Investigación Rutas de estudio y aprendizaje. Informe de coinvestigación. Trabajo de grado. Bogotá: Universidad Distrital Francisco José de Caldas.

Jara, O. (1994). Para sistematizar experiencias. San José de Costa Rica: Editorial Alforja.
Johnson, W. (1999). Los nuevos círculos del aprendizaje: La cooperación en el aula y en la escuela. Buenos Aires: Aique.

Katz, L. y Raths, J. (1985). Dispositions as goals for teacher education. En: Teaching and Teacher Education. 1 (4): 301-307.

López, A., Rodríguez, D. y Rojas, M. (2005). Condiciones en las que puede ser observada la ruta de estudio en el aula de matemáticas. Estudio exploratorio de instrumentos. Informe de coinvestigación, trabajo de grado. Investigación Rutas de estudio y aprendizaje. Bogotá: Universidad Distrital Francisco José de Caldas.

López, A., Rodríguez, D. y Rojas, M. (2005). Informe final de pasantía de investigación: Matemáticas y su aprendizaje en educación básica del distrito capital. Caracterización de las rutas de aprendizaje en el aula. Bogotá: Universidad Distrital Francisco José de Caldas.

Loughlin, C. (2002). El ambiente de aprendizaje: diseño y organización. Madrid: Ed. Morata.

Lurduy, O. y Romero J. (1999). La enseñanza de la aritmética y la formación de profesores. Bogotá: Gaia.

Lurduy, O., Sánchez, N., Rocha, P., Guerrero, F. y Gil D. (2005). Cuadernos de investigación. No. 5. Bogotá: Universidad Distrital Francisco José de Caldas.

Lurduy, O (2009). El Tetraedro Didáctico. Reflexión epistemológica en la perspectiva del estudio de las configuraciones didácticas. En prensa.

Martínez, M. (1991). La Investigación Cualitativa Etnográfica en Educación. Manual Teórico Práctico. Caracas: Trillas.

Martínez, M. (1994). La Etnografía como alternativa de investigación científica. En: Aportes: $L a$ investigación etnográfica aplicada a la educación. Bogotá: Dimensión Educativa. 
Martinic, S., Walker, H. (1987). Elementos metodológicos para la sistematización de proyectos de educación popular. Santiago de Chile: CIDE.

Maza, C. (1991). Enseñanza de la multiplicación y la división. Madrid: Editorial síntesis.

Ministerio de Educación Nacional. (1994). Ley 115. Ley General de Educación. Bogotá.

Ministerio de Educación Nacional. (1996). Resolución 2343. Diseño de lineamientos generales de los procesos curriculares del servicio público educativo y se establecen los indicadores de logros curriculares para la educación formal. Bogotá.

Ministerio de Educación Nacional. (1998). Lineamientos Curriculares para Matemáticas. Bogotá.

Ministerio de Educación Nacional. (2002). Estándares Básicos para la Calidad. Bogotá.

Ministerio de Educación Nacional. (2002). Decreto 230 (11 de febrero). Sobre normas en materia de currículo, evaluación y promoción de los educandos y evaluación institucional. Bogotá.

Morin, E, et al. (2003) Educar en la Era Planetaria. Barcelona: Editorial Gedisa.

Navarro, Pablo. (1990). Tipos de Sistemas Reflexivos. En: Antropos: Boletín de información y documentación. 22: 51-56.

Pérez, A. (2000). La cultura escolar en sociedad neoliberal. Madrid: Morata.

Pierre V.; Duval A. y Ronsin C. (1993) Una Ecología de la Escuela. Buenos Aires: Editorial Paidós.

Porlán, R. y Martín, J. (1991) El Diario del Profesor. Sevilla: Ed. Diada.

Proshansky, H., (1983). Place-identity: physical world socialization of the self. En: Journal of Environmental Psychology. 3: 57-83.
Pulecio, D., Briceño, D. y Serrano, L. (2006). Validación, diseño y pilotaje de instrumentos de recolección de información. Informe de coinvestigación. Trabajo de grado. Investigación Rutas de estudio y aprendizaje. Bogotá: Universidad Distrital Francisco José de Caldas.

Rodriguez, J. L. (1985). Curriculum, Acto Didáctico y Teoría del Texto, Madrid: Grupo Anaya.

Tourniaire, F. (1985). Proporcional reasoning: A review of the literature. En: Educational Studies in mathematics. 16: 181-204.

Universidad Distrital Francisco José de Caldas. (1999). Documento general de acreditación Licenciatura en educación básica con énfasis en matemáticas. Facultad de ciencias y educación. Bogotá

Vásquez, A. (1980). Ética. Rio de Janeiro: Ed. Civilização Brasileira.

Vergnaud, G. (1981). Quelgues orientatíons des recherches francaises en mathématiques. R.D.M. Vol. 2-3.

Vergnaud, G. (1988). Multiplicative structures. En: Hiebert J. y Behr M. (Eds.) Numbers concepts and operations in the middle grades. NCTM. Reston. VA: Erlbaum.

Vergnaud, G. (1990). Teoría de los campos conceptuales. En: Recherches en Didactiqué des $\mathrm{Ma}$ thematics. 10. (2-3): 133-170.

Vergnaud, G. (1991). El Niño, las Matemáticas y la Realidad: problemas de la enseñanza de las matemáticas. México: Ed. Trillas.

Vergnaud, G. (1996). El niño, las matemáticas y la realidad: Problemas de la enseñanza de las matemáticas en la escuela primaria. (Ortega, L. trad.) México: Trillas.

Viera, A. (1991). Matemáticas y medio. Sevilla: Diada editores 